\title{
The Nursing Counselling among Patients Undergoing Mutilating Surgery: An Experimental Study
}

\author{
Carlo De Werra ${ }^{1}$, Francesco Mangani2*, Antonio Gargiulo², Roberto Ponzo², \\ Ermelinda Gnarra², Brigida Savanelli², Francesca Romana Di Palo ${ }^{2}$ and \\ Giuseppe Galloro ${ }^{1}$ \\ ${ }^{1}$ Assistant Professor in General Surgery, Italy \\ ${ }^{2}$ Medical Intern in General Surgery, Italy
}

ISSN: 2577-2007

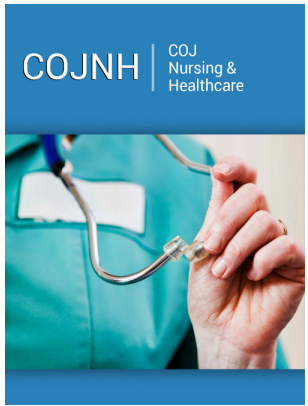

*Corresponding author: Francesco Mangani, Medical Intern in General Surgery, Italy

Submission: 价 February 17, 2020

Published: 海 February 26, 2020

Volume 6 - Issue 1

How to cite this article: Carlo De Werra, Francesco Mangani, Antonio Gargiulo, Roberto Ponzo, Ermelinda Gnarra, et al. The Nursing Counselling among Patients Undergoing Mutilating Surgery: An Experimental Study. COJ Nurse Healthcare.6(1). COJNH.000628.2020.

DOI: $10.31031 /$ COJNH.2020.06.000628.

Copyright@ Francesco Mangani, This article is distributed under the terms of the Creative Commons Attribution 4.0 International License, which permits unrestricted use and redistribution provided that the original author and source are credited.

\begin{abstract}
Background: Counselling is the psychological aspect and an adequate and sympathetic communication with the patient. It consists in making more comprehensible to the patient his situation and to facilitate him to manage it with the greatest possible autonomy.
\end{abstract}

Purpose: The purpose of our study was to evaluate the effect of the pre-operative nursing counselling in anxiety management, particularly among patients undergoing mutilating surgery.

Methods: We selected 80 patients, 43 males and 37 females, who were scheduled for either a radical colorectal or breast cancer surgery. We used a two groups comparative design with an intervention group and a control group. STAI-Y1 test was administered to both groups. We used it to evaluate the pre- and post-operative anxiety and to evaluate the effect of the counseling intervention.

Result: We calculated the preoperative and postoperative STAI-Y1 test average score for each group. There was a reduction of postoperative average score compared to preoperative one in both groups. In the intervention group, that received nursing counselling, the reduction of the postoperative average score is greater than in the control group.

Conclusion: The analysis of our data shows that nursing counselling has a very important role to reduce anxiety in the surgical patient. We hope that counselling will be a teaching subject during the nursing degree curriculum. Further studies are needed to confirm our data.

Keywords: Counselling; Anxiety; Nursing; Surgery

\section{Introduction}

Counselling is not new for the modern nurse but has ancient origins. It is the psychological aspect and an adequate and sympathetic communication with the patient. Humanistic psychology defines "Counselling" as a professional helping relationship, focused on the patient. The help consists in making more comprehensible to the patient his situation and to facilitate him to manage it with the greatest possible autonomy. We can define it as an interactive learning system that gives the patient the skills for problem management. Every healthcare provider may use interview techniques, but it can be defined "nursing counselling" if nurses learn specific skills and learn to adopt a way to conduct the interview. It is necessary that the nurses consider the knowledge that is needed to be able to pass this knowledge on to the patient, thus, defining his/her skills and activating the responses in the most appropriate time and way. Nursing counselling may provide an introduction of yourself to the patient and his/ her family as an operator able to clarify, stimulate, facilitate, encourage, provide information of the disease [1]; and these skills are not necessarily provided in the basic training [2].

Counselling has three steps: Understanding, exploration and management of the problem. The first phase is the time of reception and the first contact with the patient. The goal is to understand what the problem is. It is the clarification stage. At this stage the patient is "confused" not neurologically but subjectively. The emotional value of the problem is, for the patient, stronger and bigger than the cognitive and rational value. The task of the nurse is to encourage as much as possible to the patient in the expression of his problem, in order to allow him/her to talk and bring order to the multiplicity of data and emotions. In this first part it is useful and important to know how to listen without interfering, to understand without 
drawing conclusions and without giving personal considerations. The second step allows to redefine and clarify the issue. In the preoperative part, it's not clear to the patient what he mostly fears and what is for him the real problem; in the case of a mutilating surgery the main problems for the patients are the inevitable alteration of their physical appearance [3] and its real impact on his quality of life. Another important aspect is the fear linked to anesthesia, to complications related to the surgical operation, e.g. infections [4-6], to complications in laparoscopic surgery $[7,8]$, and to the risks of the surgery in the advanced aged patient [9]. This phase represents the moment of awareness of what is the problem. The third phase's aim is the activation of the patient's internal and external resources; therefore, it's the time of the issue management by the patient. In this phase, the goal of the nurse counsellor is to put the patient on the spot to take charge of the problem and to encourage him to find strategies to solve it.

For each of the three phases there are technical and communication skills, accentuated to help the patient to open up and trust. The nurse counselor skills do not come in the patient's psyche, in remote and unconscious dynamics; he stays with the patient and/or his family during his presence in the hospital and he/she participates in their history, without trying to change, and using skills, abilities and knowledges to resolve, as far as possible, the identified problem [10]. The purpose of our study was to evaluate the effect of the pre-operative nursing counselling in anxiety management.

\section{Materials and Methods}

We selected 80 patients, 43 males and 37 females, who were scheduled for either a radical colorectal or breast cancer surgery. Patients were 30-70 years old with a heterogeneous social-cultural status. We excluded from the sample of the study patients, those who had recently taken psychoactive drugs and patients with existing or past psychiatric disease. We used a two-group comparative design with an intervention group and a control group. Of 80 patients, 40 were placed in the intervention group who have received the nursing counselling (Group B), and the remaining 40 were placed in the control group (Group A). The characteristics of the two groups are shown in Table 1.

Table 1: Characteristics of the two groups based on age, sex and educational status.

\begin{tabular}{|c|c|c|c|c|}
\hline \multirow{2}{*}{ Characteristics } & Group A & \multirow{2}{*}{$\%$} & Group B & \multirow{2}{*}{$\%$} \\
\hline & (Control Group) & & (Intervention Group) & \\
\hline \multicolumn{5}{|c|}{ Age } \\
\hline $30-45$ & 9 & 22.5 & 11 & 27.5 \\
\hline $46-60$ & 12 & 30 & 8 & 20 \\
\hline$>60$ & 19 & 47.5 & 21 & 52.5 \\
\hline Total & 40 & 100 & 40 & 100 \\
\hline \multicolumn{5}{|c|}{ Sex } \\
\hline Male & 23 & 57.5 & 20 & 50 \\
\hline Female & 17 & 42.5 & 20 & 50 \\
\hline Total & 40 & 100 & 40 & 100 \\
\hline \multicolumn{5}{|c|}{ Education Status } \\
\hline Primary school & 5 & 12.5 & 4 & 10 \\
\hline Middle school & 7 & 17.5 & 9 & 22.5 \\
\hline High school & 25 & 62.5 & 22 & 55 \\
\hline University & 3 & 7.5 & 5 & 12.5 \\
\hline Total & 40 & 100 & 40 & 100 \\
\hline
\end{tabular}

$1=$ Not at all; $2=$ Somewhat; $3=$ moderately; $4=$ very much so. The STAI-Y1 test consists of 20 items. The total score is between 20 and 80; the threshold value of anxious symptoms is set at 40 . According to score is possible to define the severity level of anxiety: 40 to 50 mild, 50 to 60 moderate, $>60$ serious.

We informed all patients about the study and they expressed a verbal agreement to take part in this study.

STAI-Y1 test, that was developed by Spielberger et al. [11] in 1970 in the US, which was administered to both groups. STAI-Y1 test is shown in the Table 2. We used it to evaluate the pre- and post-operative anxiety. Patients filled out test after their arrival to the hospital. All patients filled out the questionnaire in about ten minutes. Then the patients in group B received an adequate nursing counselling by experienced staff. These patients received nursing counselling in the relax area of the operating room and then during the first day after operation. The nurse during the counselling gave to the patient's moral support and information about the operation and the post-operative course. $24 / 48 \mathrm{~h}$ after the operation the same STAI-Y1 test was administered again to patients of both groups. 
Table 2: STAI-Y1 test.

\begin{tabular}{|c|c|c|c|c|}
\hline Statements & Not at all & Some-what & Moderately & Very much so \\
\hline I feel calm & 1 & 2 & 3 & 4 \\
\hline I feel secure & 1 & 2 & 3 & 4 \\
\hline I'm tense & 1 & 2 & 3 & 4 \\
\hline I feel strained & 1 & 2 & 3 & 4 \\
\hline I feel tranquil & 1 & 2 & 3 & 4 \\
\hline I feel upset & 1 & 2 & 3 & 4 \\
\hline I'm presently worrying over possible misfortunes & 1 & 2 & 3 & 4 \\
\hline I feel satisfied & 1 & 2 & 3 & 4 \\
\hline I feel frightened & 1 & 2 & 3 & 4 \\
\hline I feel comfortable & 1 & 2 & 3 & 4 \\
\hline I feel self-confident & 1 & 2 & 3 & 4 \\
\hline I feel nervous & 1 & 2 & 3 & 4 \\
\hline I'm jittery & 1 & 2 & 3 & 4 \\
\hline I feel indecisive & 1 & 2 & 3 & 4 \\
\hline I'm relaxed & 1 & 2 & 3 & 4 \\
\hline I feel content & 1 & 2 & 3 & 4 \\
\hline I'm worried & 1 & 2 & 3 & 4 \\
\hline I feel confused & 1 & 2 & 3 & 4 \\
\hline I feel steady & 1 & 2 & 3 & 4 \\
\hline I feel pleasant & 1 & 2 & 3 & 4 \\
\hline
\end{tabular}

Result

Table 3: STAI-Y1 test score obtained by each patient and average score of the two groups.

\begin{tabular}{|c|c|c|c|c|c|c|c|}
\hline $\begin{array}{c}\text { Group A (Control group) } \\
\text { Pre-Operative }\end{array}$ & Score & $\begin{array}{c}\text { Group B (Intervention } \\
\text { group) } \\
\text { Pre-Operative }\end{array}$ & Score & $\begin{array}{c}\text { Group A (Control } \\
\text { group) } \\
\text { Post-Operative }\end{array}$ & Score & $\begin{array}{c}\text { Group B (Intervention } \\
\text { group) } \\
\text { Post-Operative }\end{array}$ & Score \\
\hline Pz.1A & 58 & Pz.1B & 58 & Pz.1A & 50 & Pz.1B & 50 \\
\hline Pz.2A & 67 & Pz.2B & 62 & Pz.2A & 65 & Pz.2B & 47 \\
\hline Pz.3A & 49 & Pz.3B & 47 & Pz.3A & 42 & Pz.3B & 43 \\
\hline Pz.4A & 57 & Pz.4B & 48 & Pz.4A & 52 & Pz.4B & 41 \\
\hline Pz.5A & 60 & Pz.5B & 50 & Pz.5A & 51 & Pz.5B & 42 \\
\hline Pz.6A & 58 & Pz.6B & 61 & Pz.6A & 55 & Pz.6B & 54 \\
\hline Pz.7A & 64 & Pz.7B & 59 & Pz.7A & 60 & Pz.7B & 52 \\
\hline Pz.8A & 55 & Pz.8B & 49 & Pz.8A & 45 & Pz.8B & 40 \\
\hline Pz.9A & 61 & Pz.9B & 64 & Pz.9A & 56 & Pz.9B & 52 \\
\hline Pz.10A & 55 & Pz.10B & 45 & Pz.10A & 50 & Pz.10B & 43 \\
\hline Pz.11A & 45 & Pz.11B & 52 & Pz.11A & 41 & Pz.11B & 45 \\
\hline Pz.12A & 48 & Pz.12B & 69 & Pz.12A & 40 & Pz.12B & 55 \\
\hline Pz.13A & 66 & Pz.13B & 57 & Pz.13A & 47 & Pz.13B & 43 \\
\hline Pz.14A & 59 & Pz.14B & 65 & Pz.14A & 59 & Pz.14B & 52 \\
\hline Pz.15A & 51 & Pz.15B & 55 & Pz.15A & 51 & Pz.15B & 45 \\
\hline Pz.16A & 71 & Pz.16B & 48 & Pz.16A & 60 & Pz.16B & 40 \\
\hline Pz.17A & 61 & Pz.17B & 66 & Pz.17A & 56 & Pz.17B & 51 \\
\hline
\end{tabular}




\begin{tabular}{|c|c|c|c|c|c|c|c|}
\hline Pz.18A & 57 & Pz.18B & 70 & Pz.18A & 51 & Pz.18B & 52 \\
\hline Pz.19A & 52 & Pz.19B & 57 & Pz.19A & 50 & Pz.19B & 42 \\
\hline Pz.20A & 58 & Pz.20B & 44 & Pz.20A & 53 & Pz.20B & 38 \\
\hline Pz.21A & 49 & Pz.21B & 71 & Pz.21A & 48 & Pz.21B & 58 \\
\hline Pz.22A & 55 & Pz.22B & 52 & Pz.22A & 54 & Pz.22B & 42 \\
\hline Pz.23A & 59 & Pz.23B & 65 & Pz.23A & 52 & Pz.23B & 50 \\
\hline Pz.24A & 47 & Pz.24B & 69 & Pz.24A & 45 & Pz.24B & 51 \\
\hline Pz.25A & 61 & Pz.25B & 46 & Pz.25A & 57 & Pz.25B & 36 \\
\hline Pz.26A & 66 & Pz.26B & 49 & Pz.26A & 61 & Pz.26B & 36 \\
\hline Pz.27A & 59 & Pz.27B & 50 & Pz.27A & 55 & Pz.27B & 41 \\
\hline Pz.28A & 52 & Pz.28B & 62 & Pz.28A & 47 & Pz.28B & 52 \\
\hline Pz.29A & 62 & Pz.29B & 63 & Pz.29A & 52 & Pz.29B & 49 \\
\hline Pz.30A & 57 & Pz.30B & 61 & Pz.30A & 55 & Pz.30B & 53 \\
\hline Pz.31A & 61 & Pz.31B & 70 & Pz.31A & 55 & Pz.31B & 51 \\
\hline Pz.32A & 57 & Pz.32B & 57 & Pz.32A & 54 & Pz.32B & 44 \\
\hline Pz.33A & 62 & Pz.33B & 56 & Pz.33A & 54 & Pz.33B & 43 \\
\hline Pz.34A & 55 & Pz.34B & 49 & Pz.34A & 53 & Pz.34B & 35 \\
\hline Pz.35A & 61 & Pz.35B & 51 & Pz.35A & 54 & Pz.35B & 42 \\
\hline Pz.36A & 62 & Pz.36B & 62 & Pz.36A & 58 & Pz.36B & 54 \\
\hline Pz.37A & 69 & Pz.37B & 57 & Pz.37A & 61 & Pz.37B & 43 \\
\hline Pz.38A & 55 & Pz.38B & 55 & Pz.38A & 50 & Pz.38B & 44 \\
\hline Pz.39A & 65 & Pz.39B & 57 & Pz.39A & 59 & Pz.39B & 39 \\
\hline Pz.40A & 57 & Pz.40B & 72 & Pz.40A & 53 & Pz.40B & 51 \\
\hline Average Score & 58.075 & Average Score & 57.5 & Average Score & 52.775 & Average Score & 46.025 \\
\hline S.D. & $\pm 6,0$ & S.D. & $\pm 8,0$ & S.D. & $\pm 5,6$ & S.D. & $\pm 5,9$ \\
\hline
\end{tabular}

Table 3 shows the scores on the STAI-Y1 test obtained by patients. We calculated the preoperative and postoperative average score for each group. The preoperative average scores of the two groups were roughly equivalent: 58.07 for the control group and 57.5 for the intervention group. Psychologists interpret these STAI test values as a state of moderate anxiety. The postoperative average scores of the two groups were quite different: 52.7 for the control group; 46.02 for the intervention group. There was a reduction of postoperative average score compared to preoperative average score in both groups. This reduction in both groups depends on the fact that surgery is finished, and they are out of the operating theatre. In the intervention group, that received nursing counselling, the reduction of the postoperative average score is greater than in the control group. The postoperative average score of the intervention group, according to the interpretation of STAI test, indicates a slight level of anxiety; the level of anxiety in the control group remained around moderate values.

\section{Discussion}

Between 1943 and 1954 the American psychologist Abraham Maslow conceived the concept of "Hierarchy of Needs", which identified the physical and psychological safety in the patient as a basic need immediately after the patient's physiological needs.
In 1952, Papleu with his "Interpersonal Relation in Nursing" emphasizes the relationship between nurse and patient, which is, in her opinion, the basis of nursing practice. The Theory of Dorothea Orem focuses on the patient's self-care needs. Therefore, Orem can be considered a pioneer of nursing counselling. She says that nurse's aim is to increase the patient's ability to meet his own needs independently. The Leininger's theory is based on cultural diversity and the universality. Its aim is to provide nursing to patients based on their culture. Sister Calista Roy identifies the patient as an adaptive system; according to her model, the nursing goal is to help people to adapt themselves to change and to help each individual with their psychological needs [12]. A lot of emotional problems, such as anxiety, loss of control, fear, uncertainty, decrease of selfesteem, can be experienced by surgical patients, especially for mutilating surgery, e.g. rectal cancer surgery [13-15]. A lot of trials show that the proper preparation for the surgical patient has shown success in decreasing aversive reactions; but patients, in most hospitals, don't receive an adequate education and counselling $[16,17]$. Preoperative information given to patients improves their postoperative course. Several authors have shown that when patients are well informed, they feel less anxiety and they are more mobilized. The patients want to receive information and they show greater satisfaction with the care received [18-22]. 
Anxiety represents an additional risk for the patient's health: it may interfere with his ability to learn and adapt; it may inhibit the immune and pharmacological response; it may lead to an increase in distress and post-operative pain [23]. Preoperative anxiety can affect the early prognosis, hospitalization time [24] and the consumption of postoperative analgesics [25]. Some studies have shown that an excessive state of anxiety in the patient has a negative influence for the patient's health because it causes an increased consumption of proteins by the body, a prolongation of the time of healing of surgical wounds, a potential increase in the infection risks and finally a hydro electrolytic imbalance. All these factors can affect the postoperative hospital stay, they involve more pain for the patient and a higher cost for the hospital. There are several good reasons to practice pre-operative counselling [26]. There isn't a significant correlation with the social criteria (gender, age, working conditions, education, lifestyle) and clinical criteria (diagnosis, comorbidities, type of intervention) [27]. The anxiety condition is quantified through an appropriate rating scales, including the STAI-Y test. The intervention that is more effective to reduce anxiety is counselling. Since 1975, some authors [28] observed the close relationship between the information received by the patient and his state of anxiety: they are inversely proportional.

\section{Conclusion}

Counselling is very important in the nursing of surgical patients [29]. Counselling helps patients to have an active part in their post-operative care. Effective pre-operative information helps to reduce stress, anxiety [30] and pain levels, leading to better postoperative outcomes [31-33]. The analysis of our data shows that nursing counselling has a very important role to reduce anxiety in the surgical patient. We hope that it may be used for all surgical patients, especially for patients undergoing mutilating surgery and it will be a teaching subject during the three years of the nursing degree's curriculum. Further studies are needed to confirm our data.

\section{References}

1. Raju B, Reddy K (2017) Are counseling services necessary for the surgical patients and their family members during hospitalization? Journal of Neurosciences in Rural Practice 8(1): 114-117.

2. Danon DM (2014) Counseling: The relationship that promotes personal growth. Red Edizioni, Milano, Italy.

3. De Werra C, Tramontano R, Di Filippo G, Aloia S, Di Micco R, et al. (2016) Giant lipoma in the thigh a case report. Ann Ital Chir 87:

4. De Werra C, Aloia S, Di Micco R, Del Giudice RD, Tramontano R, et al. (2015) SSIs in Italy: Prevention and surveillance during the last five years. Surgical Science 6(8): 383-394.

5. Sartelli MS, Catena FC, Ansaloni LA, Lazzareschi DV, Taviloglu K, et al. (2011) Complicated intra-abdominal infections observational European study (CIAO study). World J Emerg Surg 6(1): 40.

6. De Werra C, Del Giudice RD, Di Micco R, Aloia S, Bracciano L, et al. (2013) Biliary duct injuries in the laparoscopic era: Our experience. G Chir 34(3): 59-63.
7. De Werra C, Di Micco R, Pilone V, Formato A, Montella E, et al. (2013) Serum in vivo and in vitro activity of single dose of ertapenem in surgical obese patients for prevention of SSIs. Obes Surg 23(7): 911-919.

8. Riccio PR, De Werra C (2012) Nursing e laparoscopia: Compendio per la professione infermieristica. Forma Communications, Naples, Italy.

9. Limite G, Di Micco R, Sollazzo V, Esposito E, Cervotti M, et al. (2014) Clinically cNO breast cancer in elderly: What surgery? International Journal of Surgery 12(2): 130-134.

10. Artioli GA, Montanari RM, Saffioti AS (2004) Counseling e professione infermieristica: Teoria, tecnica, casi. Roma: Carocci Faber, Italy.

11. Spielberger CDS, Gorsuch RLG, Lushene REL (1970) State-trait anxiety inventory. Consulting Psychologist Press, Palo Alto, California, USA.

12. Manara D (2000) Verso una teoria dei bisogni dell'assistenza infermieristica. Lauri, Milano, Italy.

13. Luglio G, Sivero L, Tarquini R, D’Antonio D, Quarto G, et al. (2013) Functional results after TME for rectal cancer: J-Pouch vs coloplasty. A single institution prospective study. Chirurgia 26(4): 283-286.

14. Luglio G, Tarquini R, Sivero L, Giglio MC, De Werra C, et al. (2013) Functional and oncological outcomes after transanal local excision for rectal cancer. A prospective study. Chirurgia 26(5): 337-340.

15. Palumbo R, Del Giudice R, Cervotti M, De Filippo D, Costanzo A, et al. (2013) Escissione locale del cancro del retto sottoperitoneale dopo chemio radio terapia neoadiuvante: Valutazione della sicurezza e dell'efficacia. Antologia Italiana.

16. Asilioglu KA, Celik SSC (2004) The effect of preoperative education on anxiety of open cardiac surgery patients. Patient education and counseling 53(1): 65-70.

17. Breemhaar BB, Borne HWB, Mullen PD (1996) Inadequacies of surgical patient education. Patient education and counseling 28(1): 31-44.

18. Nelson S (1996) Pre-admission education for patients undergoing cardiac surgery. Br J Nurs 5(6): 335-340.

19. Garbee DDG, Gentry AJG (2001) Coping with the stress of surgery. AORN journal 73(5): 946-951.

20. Hathaway DH (1986) Effect of preoperative instruction on postoperative outcomes: A meta-analysis. Nurs Res 35(5): 269-275.

21. Watts S, Brooks A (1997) Patients' perceptions of the preoperative information they need about events they may experience in the intensive care unit. Journal of Advanced Nursing 26(1): 85-92.

22. De Wit P, Duivenvoorden HJ, Dixhoorn JJ (1996) More psychological preparation in heart surgery for certain patients is beneficial. Nederlands tijdschrift voor geneeskdunde 140(34): 1720-1723.

23. Wiens AGW (1998) Preoperative anxiety in women. AORN journal 68(1): 74-88.

24.Akbari M, Celik SS (2018) The effects of discharge training and post discharge counseling on quality of life after coronary artery bypass graft surgery. Nursing and Midwifery Studies 7(3): 105-110.

25.Zhang Y, Li Z, Chen J, Fan Z (2019) Effect of preoperative anxiety on early prognosis of patients after thoracoscopic lung cancer resection. Zhongguo Fei Ai Za Zhi 22(11): 714-718.

26. Boore JB (1987) Nursing the physically III adult. Churchill Livingstone, London, UK.

27. Maward LM, Azar NA (2004) Comparative study of anxiety in informed and non-informed patients in the preoperative period. Rech Soins Infirm 78: 35-58. 
28. Holmes JH (2005) Preoperative visiting: Landmarks of the journey. British journal of perioperative nursing 15(10): 434-440.

29. Nelson G, Bakkum-Gamez J, Kalogera E, Glaser G, Altman A, et al. (2019) Guidelines for perioperative care in gynecologic/oncology: Enhanced Recovery After Surgery (ERAS) Society recommendations-2019 update. International Journal Gynecological Cancer 29(4): 651-668.

30. Lemos-Neto SV, Barrucand L, Verçosa N, Tibirica E, Lemos MF (2019) Preoperative education reduces preoperative anxiety in cancer patients undergoing surgery: Usefulness of the self-reported Beck anxiety inventory. Revista Brasileira de Anestesiologia 69(1): 1-6.
31. Healy KM (1968) Does preoperative instruction make a difference? The American journal of nursing 68(1): 62-67.

32. Heather MA, Charlotte D, Mckelvie R, Hirsh J, Rush BR (2000) Effect of a preoperative intervention on preoperative and postoperative outcomes in low-risk patients awaiting elective coronary artery bypass graft surgery. A randomized, controlled trial. Annals of internal medicine 133(4): 253-262.

33. Van Aernam B, Lindeman CA (1971) Nursing intervention with the presurgical patient: The effects of structured and unstructured preoperative teaching. Nursing research 20(4): 319-332. 